Case Report

\title{
An Interstitial 20q11.21 Microdeletion Causing Mild Intellectual Disability and Facial Dysmorphisms
}

\author{
Ivan Y. Iourov, ${ }^{1,2}$ Svetlana G. Vorsanova, ${ }^{1,2,3}$ Oxana S. Kurinnaia, ${ }^{1,2,3}$ and Yuri B. Yurov ${ }^{1,2,3}$ \\ ${ }^{1}$ Mental Health Research Center, Russian Academy of Medical Sciences, Moscow 119152, Russia \\ ${ }^{2}$ Institute of Pediatrics and Children Surgery, Ministry of Health of the Russian Federation, Moscow 125412, Russia \\ ${ }^{3}$ Moscow City University of Psychology and Education, Moscow 127051, Russia
}

Correspondence should be addressed to Ivan Y. Iourov; ivan.iourov@gmail.com

Received 30 November 2012; Accepted 9 January 2013

Academic Editors: S. Ennis, S. Paracchini, J. L. Royo, and M. Velinov

Copyright (C) 2013 Ivan Y. Iourov et al. This is an open access article distributed under the Creative Commons Attribution License, which permits unrestricted use, distribution, and reproduction in any medium, provided the original work is properly cited.

We report a case of an interstitial chromosome 20q11.21 microdeletion in a 7-year-old male child presenting with mild intellectual disability and facial dysmorphisms. Array comparative genomic hybridization (CGH) has shown that the deletion resulted in the loss of 68 genes, among which 5 genes (COX4I2, MYLK2, ASXL1, DNMT3B, and SNTA1) are disease causing. The size of the deletion was estimated to span $2.6 \mathrm{Mb}$. Only three cases of deletions encompassing this chromosomal region have been reported. The phenotype of the index patient was found to resemble the mildest cases of Bohring-Opitz syndrome that is caused by ASXL1 mutations. An in silico evaluation of the deleted genomic region has shown that benign genomic variations have never been observed to affect the ASXL1 gene, in contrast to the other disease-causing genes. As a result, it was suggested that ASXL1 loss is likely to be the main cause of the phenotypic manifestations. The present case report indicates that a loss of the disease-causing gene can produce a milder phenotype of a single gene condition.

\section{Introduction}

The application of array comparative genomic hybridization $(\mathrm{CGH})$ in clinical cytogenetics has significantly increased the diagnostic yield $[1,2]$. Moreover, studying genome variations in neurobehavioral diseases using array CGH has promoted the identification of new causative submicroscopic chromosome imbalances in the clinical population $[2,3]$. As a result, array CGH molecular cytogenetic analysis has become almost indispensable in children suffering from intellectual disability and related neurobehavioral problems [1-3].

Performing a similar study in the Russian cohort of children with intellectual disability and congenital malformations (for details see [4]), we have identified an interstitial 20q11.21 microdeletion in a 7-year-old male child presenting with mild intellectual disability and facial dysmorphisms. According to the available literature, only three cases of chromosome 20 deletions encompassing the same chromosomal region (excluding somatic chromosome rearrangements associated with malignant pathology) have been reported and only two cases of interstitial deletions involving 20q11.21 were previously characterized by array CGH [5-7].

\section{Case Presentation and Methods}

2.1. Clinical Description. A 7-year-old male child was referred to molecular cytogenetic analysis, because of intellectual disability and facial dysmorphisms. He was born at 39 weeks of gestation to a 25-year-old mother and 28-year-old father. The couple is healthy and unrelated, having a history of a previous first trimester miscarriage. The pregnancy was reported to be complicated by hypertension during the third trimester. He was delivered vaginally. Neonatal measurements were as follows: birth weight was $2.9 \mathrm{~kg}$ (10th centile), and length was $49 \mathrm{~cm}$ (25th centile). At the age of one week, feeding problems (feeding intolerance and food refusal) were noted. Physical examination made at the age of 7 years showed microretrognathia, hypertelorism, upslanting palpebral fissures, prominent eyes, broad nasal bridge, low set ears, and 


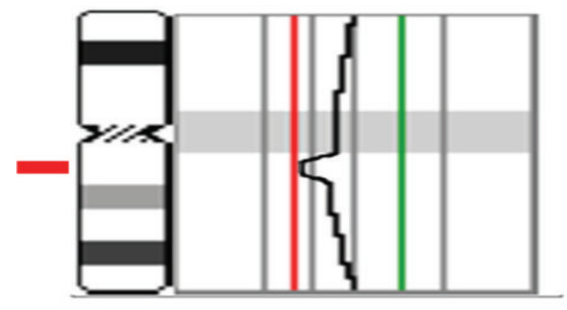

(a)

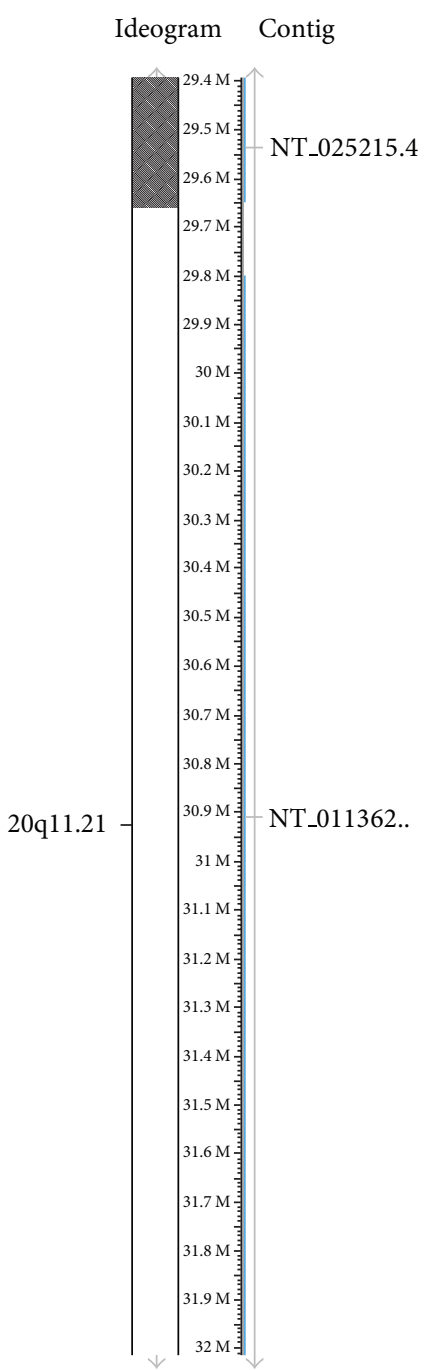

Genes seq Symbol

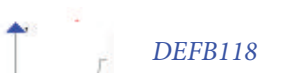

DEFB119

REM1

HM13

ID1

COX4I2

$B C L 2 L 1$

TPX2

MYLK2

PDRG1

XKR7

HCK

PLAGL2

POFUT1

KIF3B

ASXL1

C20orf203

DNMT3B

MAPRE1

EFCAB8

SUN5

BPIFB2

BPIFB3

BPIFA2

BPIFA4P

BPIFA1

BPIFB1

BPIFB5P

CDK5RAP1

SNTA1

Region displayed: 29.39 K-32.02 bp

Total genes on chromosome: 897

Genes labeled: 30, total genes in region: 68

(c)

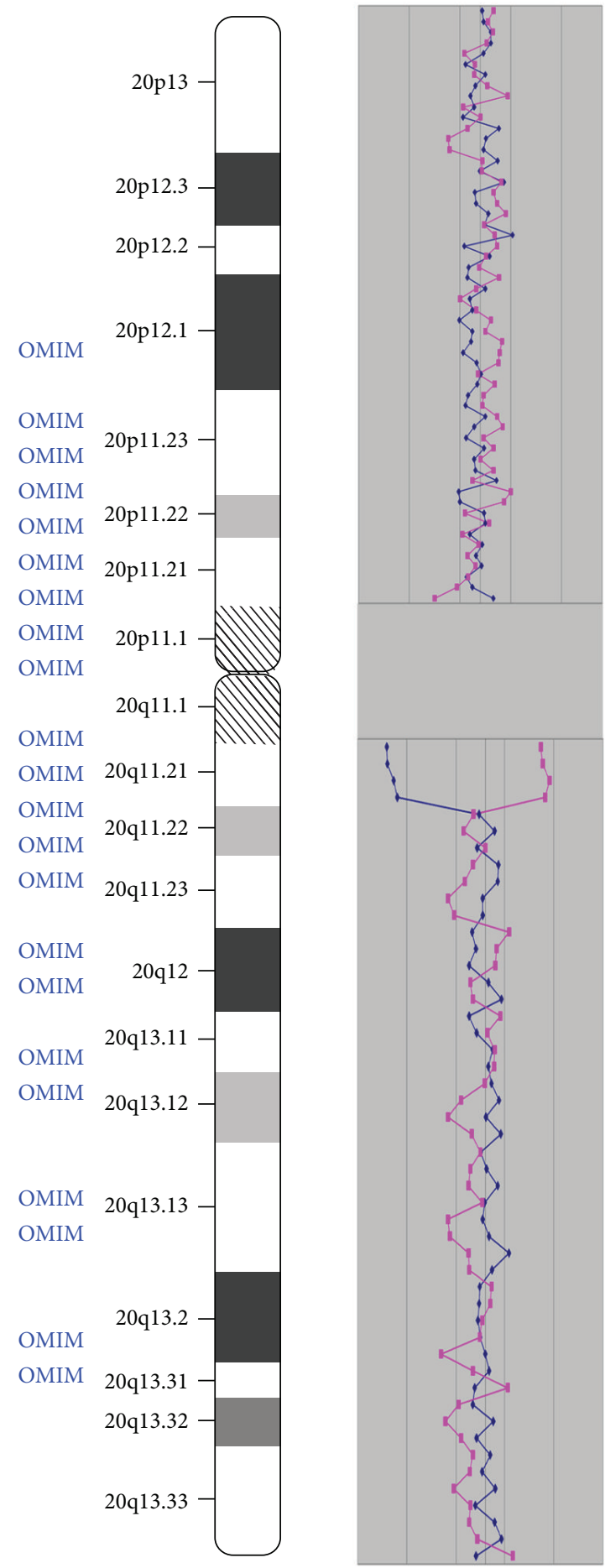

(b)

FIGURE 1: Molecular cytogenetic (CGH) findings in the index case: (a) high-resolution metaphase CGH demonstrating ish cgh $\operatorname{dim}(20)$ (q11.21q11.21); (b) array CGH demonstrating arr 20q11.21(29,392,835-32,017,043)x1 (two alternative arrays Cy3/Cy5 (pink line) and Cy5/Cy3 (blue line) were plotted on the graph); (c) and depiction of the deleted chromosomal region by NCBI Build 37.1/NCBI Map Viewer (http://www.ncbi.nlm.nih.gov/projects/mapview/map_search.cgi?taxid=9606). 
low frontal hairline. Mild intellectual disability and speech delay were noticed.

2.2. Cytogenetic Analysis. Cytogenetic analysis was performed by GTG-banding according to standard procedures. Thirty metaphase plates were studied at a resolution higher than 550 bands. Detectable karyotype abnormalities were not found.

\subsection{High-Resolution Metaphase CGH. High-resolution} metaphase CGH was performed according to previously described protocols of DNA labeling, hybridization, and detection $[8,9]$. The use of this technique has suggested the presence of a deletion in 20q11.2 (Figure 1(a)).

2.4. Array CGH. Array CGH was done using the customized human genomic microarrays (slightly modified Constitutional Chip 4.0) containing about 5000 human BAC/PAC clones (Human BAC Array-System, Perkin Elmer, USA) achieving a resolution of $0.3-1 \mathrm{Mb}$ for the whole genome scan. Technical performance of array CGH (DNA labeling, hybridization, detection, and data analysis) was made according to previously described protocols $[4,10,11]$ and to manufacturers' instructions. Array CGH has revealed an interstitial deletion in 20q11.21 spanning from 29,392,835 to $32,017,043$ (confirmed by four BAC probes: RP5-1018D12, RP5-836N17, RP5-1085F17, and RP5-1125A11 in two reverse assays). The minimal deletion size was estimated to be about $2.6 \mathrm{Mb}$ (Figure $1(\mathrm{~b})$ ).

2.5. In Silico Evaluation of the Deleted Chromosomal Region. To get further insights into understanding of the phenotypic outcome, we evaluated the deleted region by NCBI Build 37.1 (Figure 1(c)) and UCSC Genome Browser (Figure 2) as previously described [4]. The deletion resulted in the loss of 68 genes, among which 23 genes are listed in OMIM (http://www.omim.org/). Among the latter, five genes are disease causing: COX4I2 (exocrine pancreatic insufficiency, dyserythropoietic anemia, and calvarial hyperostosis/OMIM: 612714), MYLK2 (cardiomyopathy, hypertrophic, midventricular, and digenic/OMIM: 192600), ASXL1 (Bohring-Opitz syndrome/OMIM: 605039 and myelodysplastic syndrome, somatic/OMIM: 614286), DNMT3B (immunodeficiencycentromeric instability-facial anomalies syndrome 1 or ICF syndrome/OMIM: 242860), and SNTA1 (long QT syndrome 12/OMIM: 612955). It is noteworthy, that no similar cases are indexed in DECIPHER (database of unbalanced chromosome aberrations http://decipher.sanger.ac.uk/) and benign genome variations (retrieved from Database of Genomic Variants http://dgvbeta.tcag.ca/dgv/app/home?ref=GRCh37/ hg19) mapped to this genomic region involve DNMT3B (Figure 2).

\section{Discussion}

We present a case of an interstitial microdeletion $(2.6 \mathrm{Mb})$ in $20 \mathrm{q} 11.21$ that has resulted in a loss of 68 genes. The chromosomal region was previously reported to be deleted in three cases (deletions of $6.5,6.6$, and $6.8 \mathrm{Mb}$ within 20q11.2-q12) [5-7]. Generally, interstitial deletions of the long arm of chromosome 20 are rare. Apart from the previously mentioned three cases, another ten cases of intellectual disability (mental retardation) and congenital anomalies were associated with deletions within the long arm of human chromosome 20 (for more details see [7]). Clinically, cases of deletions in chromosome 20q11.2-q12 are similar to the present one. However, the index case exhibits a significantly milder phenotype compared to previous ones. For instance, intellectual disability and facial dysmorphisms were all more severe in cases demonstrating deletions of 20q11.2q12. Interestingly, all the facial dysmorphisms and feeding problems are observed not only in cases of 20q11.2-q12 loss, but also in Bohring-Opitz syndrome. The latter condition is usually the result of mutations in ASXL1 [12]. To be more precise, addressing the study of Hoischen and colleagues [12], we have noticed that feeding problems, microretrognathia, hypertelorism, upslanting palpebral fissures, prominent eyes, broad nasal bridge, low set ears, and low frontal hairline are all observed in ASXL1 mutation positive cases. On the other hand, patients with Bohring-Opitz syndrome usually exhibit much more severe phenotype as to the index case (i.e., severe (profound) intellectual disability; growth retardation; and craniofacial, ophthalmic, and neurological abnormalities). The phenotypic differences can be explained by a suggestion that ASXL1 mutations cause loss of functions, whereas an allelic loss is likely to result in ASXL1 dosage decrease that is probably less severe if the gene is not mutated.

Apparently, phenotypic manifestations of diseases caused by genomic variations within the remaining four genes were not observed in the present case. In this context, one can propose that an allelic loss of these genes can be benign and is likely to be observed in unaffected individuals. In silico evaluation of the deleted genomic region has shown that benign genomic variations have never been observed to affect the ASXL1 gene, in contrast to the other disease-causing genes within this chromosomal region. Moreover, the loss of DNMT3B, mutations in which cause immunodeficiencycentromeric instability-facial anomalies syndrome, appears not to contribute to the present phenotype. On the other hand, benign variations encompassing this gene have been reported (Figure 2) allowing a suggestion that DNMT3B allelic losses can be benign in some cases. Taking into account the data acquired by in silico analysis, we have proposed that ASXL1 loss is the main cause of phenotypic abnormities in this case. We have to add that the presence of an ASXL1 mutation is unlikely in the index case according to comparative analysis between Bohring-Opitz syndrome and 20q11.21 microdeletion phenotypes. Thus, the present case might be considered as an example of nonmutated ASXL1 loss, which is likely to be associated with milder phenotypes of Bohring-Opitz syndrome. Nonetheless, the loss of genes, which are not associated with a specific disease, can contribute to the phenotype, as well. Finally, somatic mosaicism, which reduces the consequences of genomic imbalances and is a relatively frequent occurrence among structural chromosome abnormalities [13], can be theoretically considered as a potential cause of mild phenotypic manifestations. In 


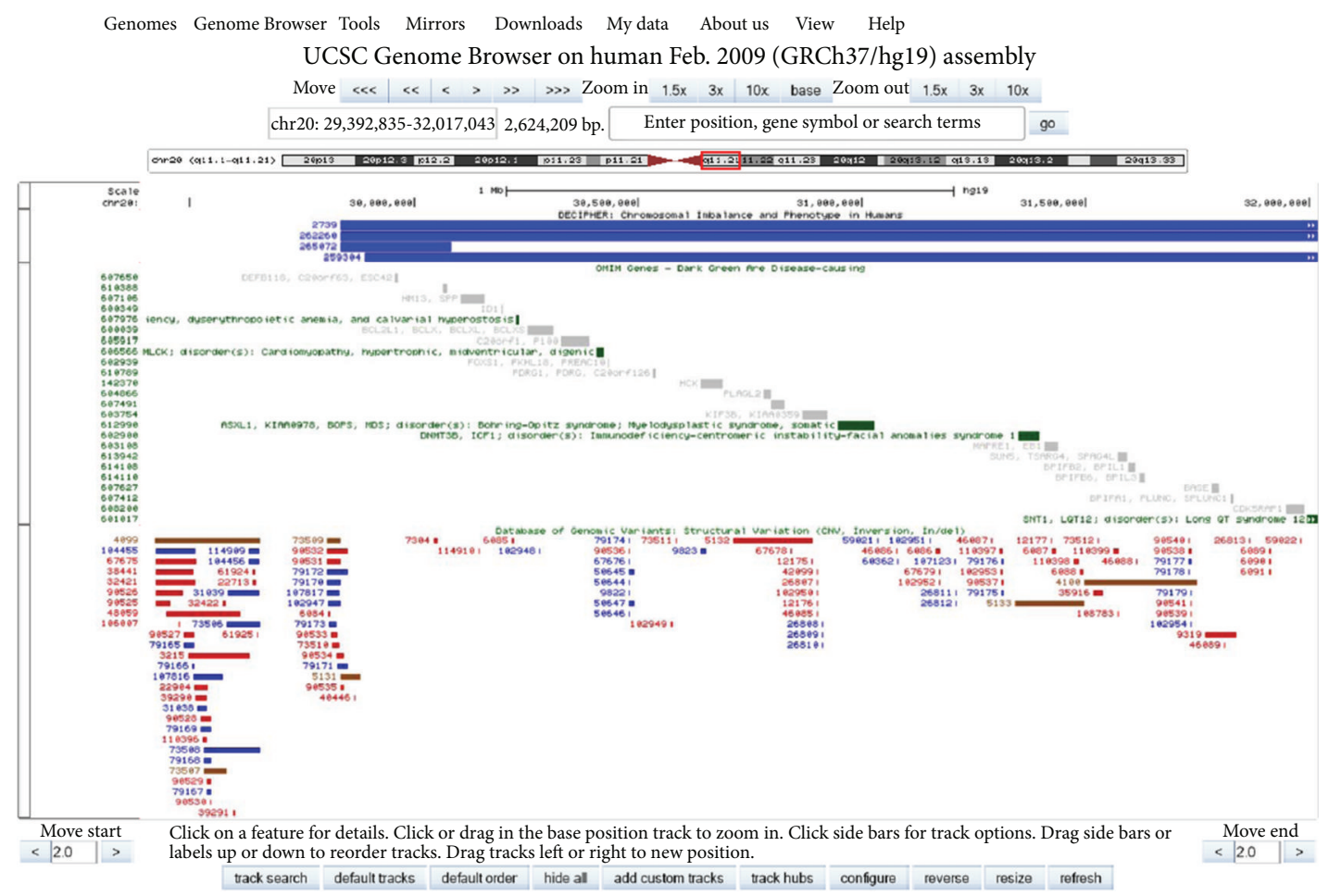

FIGURE 2: In silico evaluation of the deleted region by UCSC Genome Browser (GRCh37/hg19) (http://genome.ucsc.edu/), including data retrieved from DECIPHER (database of unbalanced chromosome aberrations http://decipher.sanger.ac.uk/), OMIM (http://www.omim.org/) (dark green OMIM genes are disease causing), and Database of Genomic Variants (http://dgvbeta.tcag.ca/dgv/app/home?ref=GRCh37/hg19) (blue bars correspond to a gain in size relative to the reference; red bars correspond to a loss in size relative to the reference; brown bars correspond to both losses and gains in size relative to the reference.).

conclusion, the present case report indicates that a deletion can lead to a milder phenotype of a single gene condition and the loss of neighboring disease-causing genes due to the same deletion can be benign. To our knowledge, this is the first case of 20 q11.21 microdeletion that is associated with such a mild phenotype.

\section{Disclosure}

In the present work, modified Constitutional Chip 4.0 was applied. It was purchased from the local Perkin Elmer agency at the time when no other microarray companies were present at the local market. We have not ever received any funding or promotion from the Perkin Elmer. None of the authors has been ever involved in the sale and distribution. Therefore, we do not have a direct financial relation with the commercial identity mentioned in our paper and do not have any conflict of interests.

\section{Acknowledgments}

Dr. IY Iourov is supported by the Grant of the President of the Russian Federation MD-4401.2013.7.

\section{References}

[1] L. G. Shaffer and B. A. Bejjani, "Medical applications of array CGH and the transformation of clinical cytogenetics," Cytogenetic and Genome Research, vol. 115, no. 3-4, pp. 303-309, 2006.

[2] B. A. Bejjani and L. G. Shaffer, "Clinical utility of contemporary molecular cytogenetics," Annual Review of Genomics and Human Genetics, vol. 9, pp. 71-86, 2008.

[3] I. Y. Iourov, S. G. Vorsanova, and Y. B. Yurov, "Molecular cytogenetics and cytogenomics of brain diseases," Current Genomics, vol. 9, no. 7, pp. 452-465, 2008.

[4] I. Y. Iourov, S. G. Vorsanova, O. S. Kurinnaia, M. A. Zelenova, A. P. Silvanovich, and Y. B. Yurov, "Molecular karyotyping by array CGH in a Russian cohort of children with intellectual disability, autism, epilepsy and congenital anomalies," Molecular Cytogenetics, vol. 5, no. 1, article 46, 2012.

[5] P. Calllier, L. Faivre, N. Marle et al., "Major feeding difficulties in the first reported case of interstitial 20q11.22-q12 microdeletion and molecular cytogenetic characterization," The American Journal of Medical Genetics A, vol. 140, no. 17, pp. 1859-1863, 2006.

[6] M. A. Iqbal and M. Al-Owain, "Interstitial del(20)(q11.2q12)clinical and molecular cytogenetic characterization," The American Journal of Medical Genetics A, vol. 143, no. 16, pp. 18801884, 2007. 
[7] Y. Hiraki, A. Nishimura, M. Hayashidani et al., "A de novo deletion of 20q11.2-q12 in a boy presenting with abnormal hands and feet, retinal dysplasia, and intractable feeding difficulty," The American Journal of Medical Genetics A, vol. 155, no. 2, pp. 409414, 2011.

[8] I. Y. Iourov, S. G. Vorsanova, E. A. Kirillova, and Y. B. Yurov, "First case of $\operatorname{del}(1)(\mathrm{p} 36.2 \mathrm{p} 33)$ in a fetus delivered stillborn," Prenatal Diagnosis, vol. 26, no. 11, pp. 1092-1093, 2006.

[9] M. Kirchhoff, H. Rose, and C. Lundsteen, "High resolution comparative genomic hybridisation in clinical cytogenetics," Journal of Medical Genetics, vol. 38, no. 11, pp. 740-744, 2001.

[10] D. Caserta, M. Benkhalifa, M. Baldi, F. Fiorentino, M. Qumsiyeh, and M. Moscarini, "Genome profiling of ovarian adenocarcinomas using pangenomic BACs microarray comparative genomic hybridization," Molecular Cytogenetics, vol. 1, article 10, 2008.

[11] M. L. Slovak, D. D. Smith, V. Bedell et al., "Assessing karyotype precision by microarray-based comparative genomic hybridization in the myelodysplastic/myeloproliferative syndromes," Molecular Cytogenetics, vol. 3, no. 1, article 23, 2010.

[12] A. Hoischen, B. W. M. van Bon, B. Rodríguez-Santiago et al., "De novo nonsense mutations in ASXL1 cause Bohring-Opitz syndrome," Nature Genetics, vol. 43, no. 8, pp. 729-731, 2011.

[13] I. Y. Iourov, S. G. Vorsanova, and Y. B. Yurov, "Somatic genome variations in health and disease," Current Genomics, vol. 11, no. 6, pp. 387-396, 2010. 


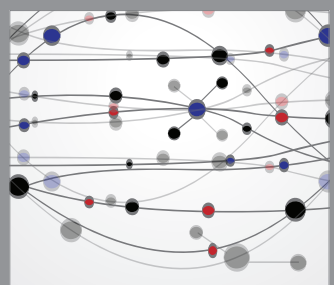

The Scientific World Journal
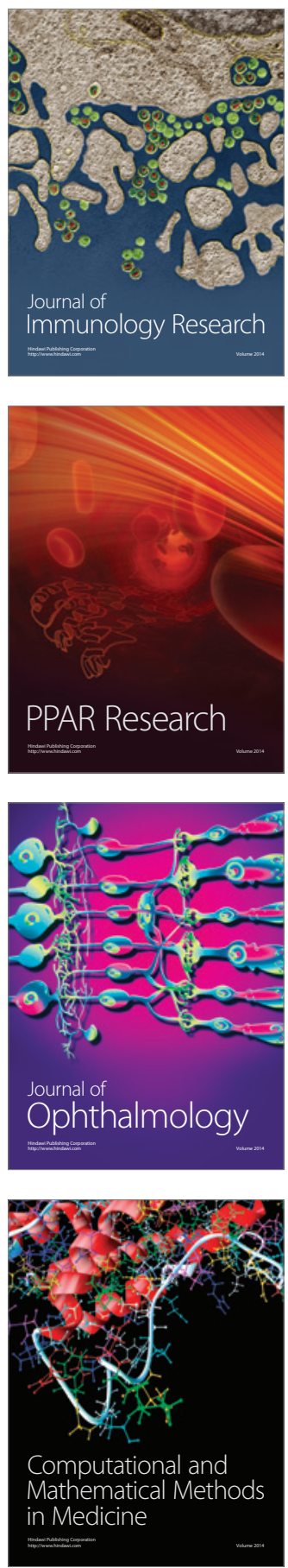

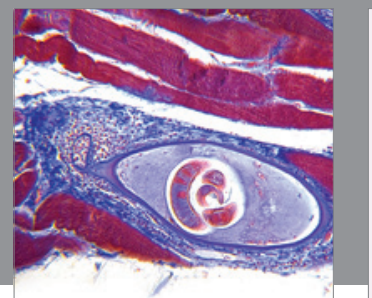

Gastroenterology

Research and Practice
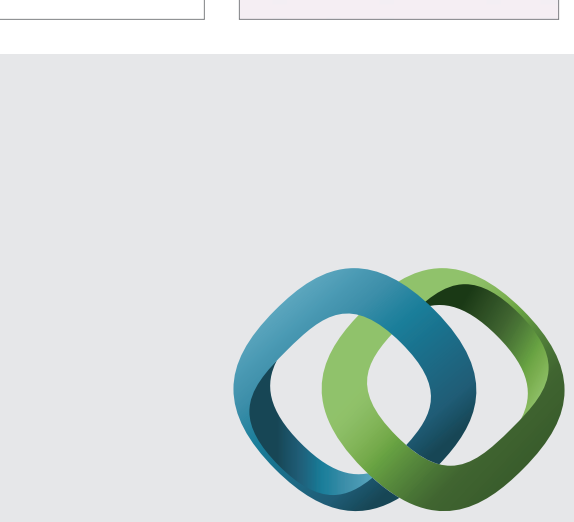

\section{Hindawi}

Submit your manuscripts at

http://www.hindawi.com
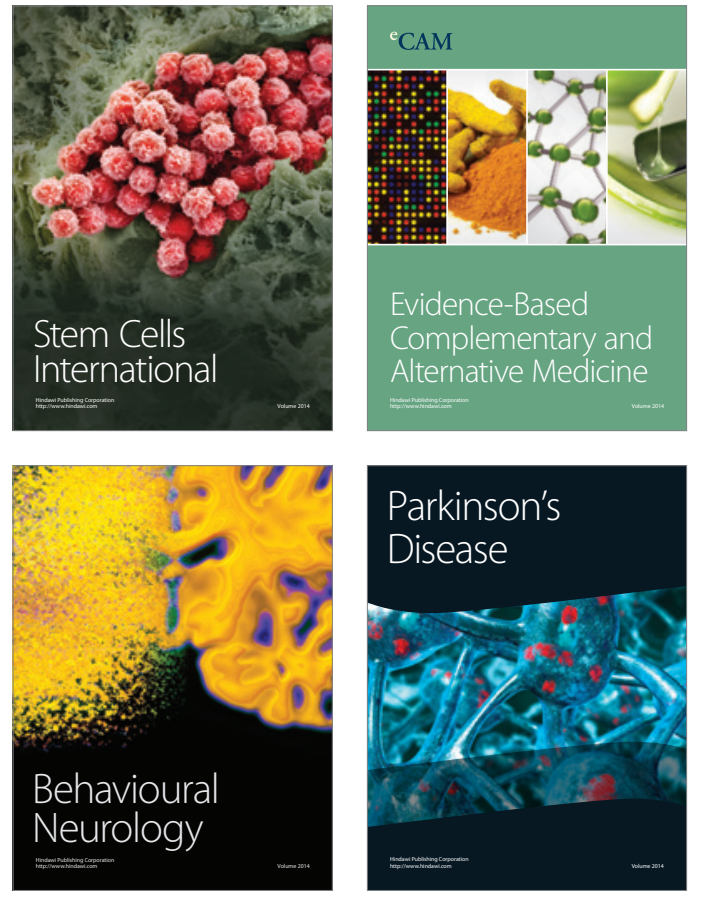
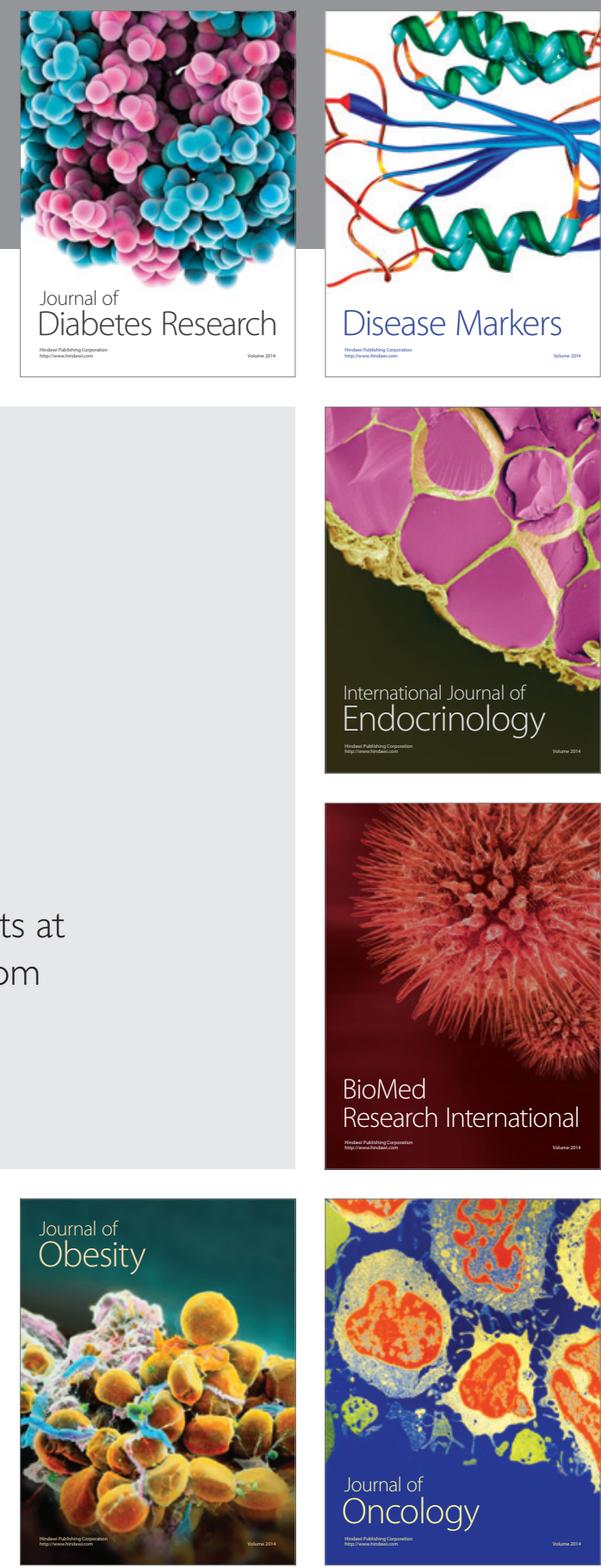

Disease Markers
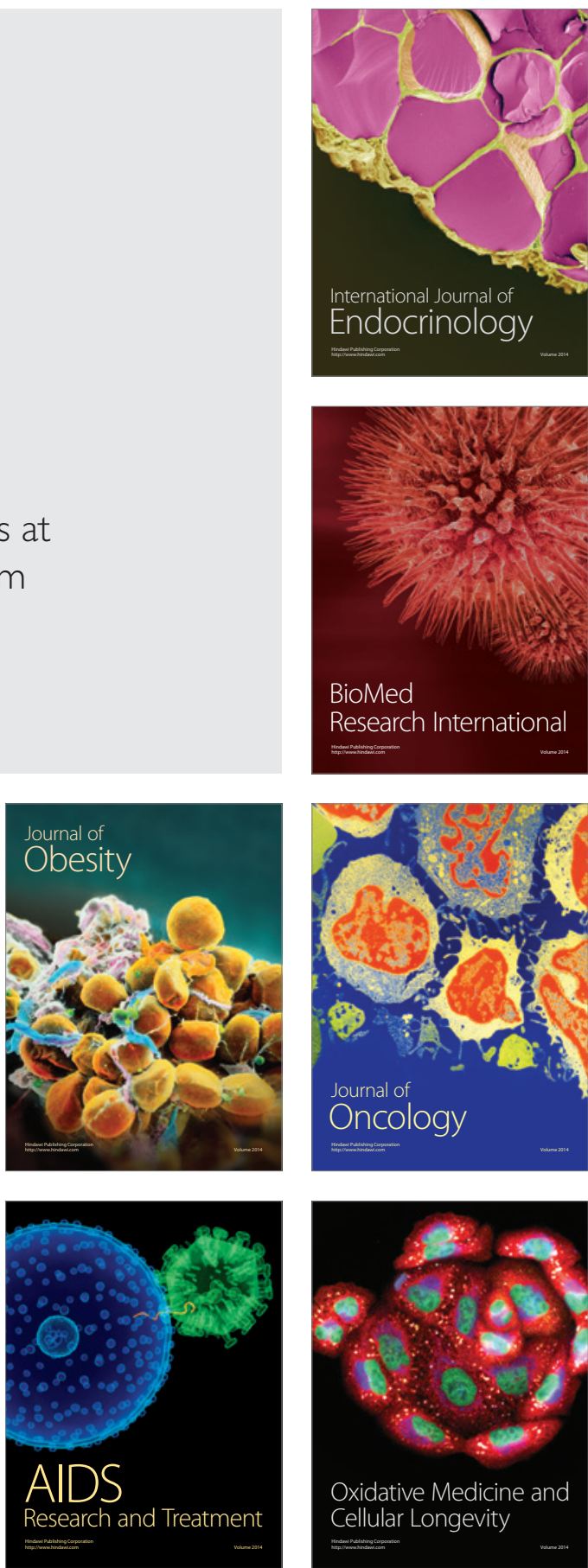